\title{
Guideline
}

\section{GUIDELINES ON FIBRINOGEN ASSAYS}

Haematology departments in the UK have traditionally performed fibrinogen assays to detect decreased levels and abnormalities of fibrinogen, and to assess haemorrhagic risk. It has also been shown that elevated fibrinogen levels are a predictor of a variety of arterial cardiovascular events, and fibrinogen assays are sometimes recommended with this in mind.

The Clauss fibrinogen assay (based on the thrombin clotting time) is the most popular technique in UK hospital laboratories, although many other methods are also in use. There appears to be great variability in both the source of reagents and the exact method used for the Clauss assay. Most laboratories are now equipped with automated coagulation analysers, and many of these perform a fibrinogen estimation derived from the degree of change of light scatter or optical density during the prothrombin time (PT-Fg). A number of problems have been described in the use of the PT-Fg method; it generally gives higher values than the Clauss technique, but the exact degree of discrepancy seems to depend on a number of different variables. International and National standards are available for fibrinogen, but do not appear to be universally used.

These guidelines have been prepared against this background and recommend which methods should be used in various clinical settings, as well as highlighting a variety of problems with fibrinogen assays.

\section{INTRODUCTION}

Fibrinogen is a major plasma protein (normal concentration $1 \cdot 5-4.0 \mathrm{~g} / \mathrm{l})$, which is synthesized in the hepatocyte. It comprises two each of three different polypeptide chains $(\mathrm{A} \alpha, \mathrm{B} \beta$ and $\gamma)$ linked by disulphide bridges. Thrombin cleaves the $A \alpha$ and $B \beta$ chains to release fibrinopeptides $A$ and $\mathrm{B}$, respectively, from the amino-terminal ends. After the fibrinopeptides are released, the resulting fibrin monomers undergo polymerization to form an insoluble fibrin clot (Hantgan et al, 1994). Fibrinogen also shows heterogeneity due to cleavage in the circulation, at the carboxyl termini of the polypeptide chains, giving rise to a series of molecules of varying size (de Maat et al, 1999). Fibrinogen and fibrin can be degraded to progressively smaller fragments by proteolytic enzymes, including plasmin and neutrophil elastase.

Fibrinogen is an acute phase reactant (Whicher \& Dieppe, 1985; Hantgan et al, 1994) and levels may be raised in relation to a variety of physiological variables and inflammatory conditions (Table I). The variation of fibrinogen

Correspondence: The Secretary, British Society for Haematology, 2 Carlton House Terrace, London SW1Y 5AF, UK. levels within the same normal subject has been observed to be approximately $1.00 \mathrm{~g} / \mathrm{l}$ in a study of 14 normal subjects over a 3-year period, although in some of the subjects fibrinogen levels varied by more than $2.5 \mathrm{~g} / \mathrm{l}$ (Thompson et al, 1987). Such variation must be taken into account when fibrinogen is being considered as a risk factor for ischaemic heart disease. One further pre-analytical variable that has been reported to affect fibrinogen measurements, which is relevant to epidemiological studies, is the season of the year; fibrinogen levels are considerably higher in winter (van der Bom et al, 1997). A seasonal difference of $0.34 \mathrm{~g} / \mathrm{l}$ $(95 \%$ CI $0 \cdot 29-0 \cdot 30 \mathrm{~g} / \mathrm{l})$ was present in subjects aged $55-75$ years which was more pronounced at age $>75$ years.

A polymorphsim of the beta fibrinogen gene promoter (G-455A) has been associated with increased levels of fibrinogen (Humphries et al, 1999). Carriers of the A-allele (about $20 \%$ of the population) have $7-10 \%$ higher fibrinogen levels than those of the GG genotype. Reduced fibrinogen levels may occur as a result of inherited gene defects, resulting in abnormal, reduced or absent fibrinogen protein, or as a result of liver disease or consumptive coagulopathies.

\section{THE ROLE OF FIBRINOGEN ASSAYS}

Traditionally, fibrinogen assays have been required in the investigation of haemorrhagic states or in laboratory testing for haemorrhagic disorders (Table II). Some centres use a fibrinogen assay along with the prothrombin time (PT) and activated partial thromboplastin time (APTT), as part of a general haemostatic screen. In this situation, the fibrinogen level replaces the use of a thrombin clotting time, but the information provided may differ depending on the type of assay used. Whereas a sensitive thrombin time will detect low fibrinogen levels, decreased fibrin monomer polymerization, abnormal fibrinopeptide release, and may be influenced by acquired dysfibrinogens, heparin or fibrin/ fibrinogen degradation products (FDP's), different fibrinogen assays show varying sensitivity to each of these factors.

\section{Inherited fibrinogen defects}

The rare conditions of congenital afibrinogenaemia, hypofibrinogenaemia and dysfibrinogenaemia are due to gene defects, resulting in reduced fibrinogen synthesis and release and/or abnormal molecular species. In afibrinogenaemia, there is a grossly decreased hepatic synthesis of fibrinogen, with very low or undetectable plasma fibrinogen, which results in a haemorrhagic diathesis, with prolonged clotting times and abnormal platelet function. In hypofibrinogenaemia, circulating fibrinogen levels show a mild-to-moderate 
Table I. The physiological, pathological and lifestyle factors influencing fibrinogen level.*

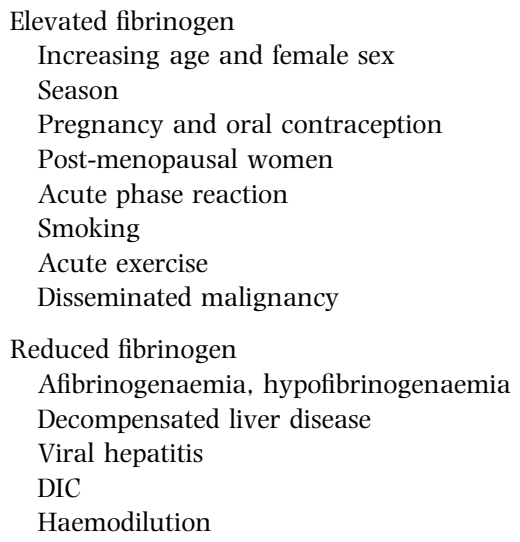

*(Eliasson et al, 1993; Hantgan et al, 1994; Lowe et al, 1997; Kluft \& Lansink, 1997; van der Bom et al, 1997; Humphries et al, 1999).

Table II. The clinical utility of fibrinogen assays.

Investigation of a haemorrhagic state (afibrinogenaemia, hypofibrinogenaemia, acquired or congenital dysfibrinogenaemia)

Investigation of unexpected prolonged/abnormal coagulation tests Detection of DIC

The establishment and monitoring of prolonged thrombolytic therapy

During Arvin therapy

Risk assessment profiling for arterial disease

Commercial use (diagnostics industry, pharmaceutical industry, blood transfusion service) for quality monitoring and potency assignment (e.g. factor VIII and fibrinogen concentrates)

reduction, and patients may be asymptomatic or have haemorrhagic problems. Dysfibrinogenaemia is characterized by abnormal fibrinogen function, therefore, immunoassays tend to give disproportionately higher levels than clottable protein measurements. In some patients, hypodysfibrinogenaemia is present (reduced levels of fibrinogen protein and abnormal function). Approximately 250 patients with dysfibrinogenaemia have been reported in the literature, $55 \%$ of whom were asymptomatic, $25 \%$ of whom had a haemorrhagic tendency and $20 \%$ of whom had thrombophilia (Haverkate \& Samama, 1995). The precise incidence of inherited dysfibrinogenaemia is unclear, as patients are usually detected because of a prolonged thrombin clotting time and obviously this will only be sensitive to certain types of fibrinogen abnormality.

\section{Acquired fibrinogen defects}

Acquired dysfibrinogens are usually due to excessive glycosylation of the fibrinogen molecule and typically occur in liver disease. In addition, in hepatocellular failure, fibrinogen levels may be markedly reduced and fibrinogen function may be influenced by the presence of elevated levels of FDPs. In disseminated intravascular coagulation (DIC), there is gross consumption of fibrinogen due to generalized fibrin formation throughout the microvasculature, and the presence of a variety of degraded forms of fibrinogen and FDPs. Massive blood transfusion or the use of colloids may also result in decreased fibrinogen levels as a result of haemodilution.

Systemic thrombolytic therapy results in a gross reduction in fibrinogen levels and there is a high risk of haemorrhage. Prior to starting a prolonged infusion (over more than $1 \mathrm{~d}$ ) of a plasminogen activator, and serially after starting therapy, the fibrinogen level may be monitored as an indication of appropriate therapy (target $0 \cdot 5-1 \cdot 0 \mathrm{~g} / \mathrm{l}$; Ludlam et al, 1995). In some patients receiving thrombolytic therapy, a fall in fibrinogen does not occur and an adjustment of therapy is necessary to prevent venous thrombotic extension (Marder \& Sherry, 1988).

\section{Increased fibrinogen}

Elevated fibrinogen levels are also clinically relevant. Fibrinogen is a major plasma protein and, therefore, a small elevation in fibrinogen level will have a significant impact on plasma viscosity, and thereby modify blood rheology. Increased plasma viscosity (e.g. in hyperviscosity syndrome) has been associated with an increased risk of thromboembolism. In recent years, a number of large prospective studies have shown that the fibrinogen level is a predictor of a variety of cardiovascular events, including stroke, myocardial infarction, leg ischaemia (in arterial disease) and postsurgical arterial re-occlusion (Lowe \& Rumley, 1999). This statistical association has remained significant regardless of the type of fibrinogen assay employed, although some authors have suggested that immunological assays may be better predictors of cardiovascular disease than functional assays (Cremer et al, 1994; Sweetnam et al, 1996, 1998). However, the interpretation of a fibrinogen value at the upper end of the normal range, or of an elevated fibrinogen measurement, in an individual patient is often difficult because of the genetic, physiological and other factors outlined above (Machin \& Mackie, 1993). It is not known whether all types of fibrinogen assay are equally sensitive to the increased fibrinogen levels resulting from these different conditions. The various fibrinogen assays may have a quite different sensitivity and clinical utility when used on an individual patient basis.

\section{THE MEASUREMENT OF FIBRINOGEN}

A variety of different tests and assays of fibrinogen have been used by clinical laboratories (for review see de Maat et al, 1999). The assays vary in the degree of expertise and time required, and the equipment available. Some tests are designed for emergency situations, where an exact fibrinogen level may not be required but merely an estimate of whether the levels are normal or grossly decreased. The expanded range of coagulation automation and reagents has had a varying impact on the performance of laboratory 
tests and their precision. Automated coagulometers utilize different technologies to detect fibrin clot end-points. Some employ mechanical principles involving the movement of a metal hook or clot impedance of a ball bearing in a magnetic field; others use photo-optical devices to measure changes in light transmission or light scatter through $90^{\circ}$. Coagulation analysers that use a photo-optical detection principle continuously monitor the change in optical density or light scatter and the degree of change is proportional to the fibrinogen concentration: hence the derived fibrinogen assay. The most reliable derived fibrinogen methods are based on the prothrombin time (PT-Fg), where there is rapid thrombin generation and fibrin formation.

\section{TYPES OF FIBRINOGEN ASSAY}

Clauss assays

A high concentration of thrombin (ranging from 35 to $200 \mathrm{U} / \mathrm{ml}$, but typically about $100 \mathrm{U} / \mathrm{ml}$ ) is added to dilute test plasma and the clotting time is measured (Clauss, 1957). The test result is compared with a calibration curve prepared by clotting a series of dilutions of a reference plasma sample of known fibrinogen concentration, and a result in $\mathrm{g} / \mathrm{l}$ is obtained. The technique is relatively time consuming and requires a high degree of technical expertise if performed by manual methods, as the end-point is very difficult to determine at the higher dilutions of standard plasma, in test samples with low fibrinogen content, or in samples forming friable clots. Mechanical end-point techniques depend on the tensile strength of the clot and, although this makes them sensitive at low fibrinogen concentrations, they are particularly affected by heparin therapy. Photo-optical systems depend on the change in optical density resulting from fibrin formation, which decreases light transmission and increases scattered light. These methods are usually suitable for detecting weak fibrin formation, but may give erroneous values when fibrin monomer polymerization is slow. They are often affected by turbid or lipaemic plasmas, while the light transmission may be affected by bile pigment and free haemoglobin, depending on the wavelength used by the optical system.

Most laboratories now use commercial reagents and an automated method, and a multiplicity of products are available, varying in thrombin strength, buffer composition (inclusion of inhibitors of heparin and FDP's), calibration method and dilution range. The reagents in common use in the UK have been evaluated (MDA, 2000; Mackie et al, 2002) and only small differences in fibrinogen levels were observed. There were significant differences in Clauss estimations depending on the analyser used, but these are unlikely to have a clinical impact $(\sim 0 \cdot 1 \mathrm{~g} / \mathrm{l})$. A reasonable degree of precision was obtained with normal plasma [coefficient of variation (CV) typically $3-7 \%$ by optical methods and $6-9 \%$ by mechanical]. The performance of Clauss fibrinogen assays by fully automated coagulometers in routine clinical laboratories may decrease the throughput for samples requiring only $\mathrm{PT}$ and APTT, and many laboratories only perform them when there is a special indication. There is also the potential on some analysers for reagent carry-over, as the Clauss assay uses a high concentration of thrombin, which has the propensity of sticking to foreign surfaces and may contaminate subsequent tests (e.g. PT, APTT), resulting in a false shortening of clotting times.

The standard plasma or calibrant must be carefully selected, as very turbid reference plasmas may cause poor linearity on photo-optical coagulometers and poor parallelism with serial dilutions of test plasmas (MDA, 1999). This may lead to incorrect potency assignment, particularly for samples with low fibrinogen levels, when single-point assays are performed. Some commercial reference plasmas designed for use in Clauss assays, appear to be erroneously calibrated when compared with the International Standard. This may reflect the calibration method used by the manufacturer or the use of calibrants other than the International Standard.

\section{Recommendations}

Although there is generally little impact of reagent system or analyser on the Clauss assay, certain clinical subgroups (e.g. DIC, thrombolytic therapy) may show greater discrepancies, probably as a result of the thrombin concentration and buffer composition (which will influence the impact of heparin and FDP's). Where possible, assays should not be performed on samples collected within $4 \mathrm{~h}$ of administration of therapeutic doses of unfractionated heparin, or on samples collected from heparin-contaminated venous or arterial lines. This may be a particular problem during cardiopulmonary bypass or renal dialysis procedures, and in these situations it may be necessary to remove the heparin using suitable ion exchange resins or heparinase enzymes.

The reagent must only be used during its stated period of stability, and care should be taken that $\mathrm{pH}$ changes do not occur as a result of the reagent vial remaining open to the air for prolonged periods. If single calibration curves are used for the lifespan of the reagent, ensure that the reagent is still stable/viable for the entire period. Quality control samples should be used to check for temporal drift. As most Clauss reagents contain high concentrations of thrombin, appropriate cleaning methods should be employed on the reagent delivery system of the analyser to avoid carry-over.

An optically clear fibrinogen standard is desirable to avoid loss of linearity on some analysers. A suitable dilution range must be used (this will vary depending on the calibrant, reagent and analyser) and the calibration curve must be linear (this normally requires logarithmic transforms). The calibration curve must include at least three (but preferably five) dilutions of the plasma, and duplicate clotting times are advised. Test samples should be re-diluted if they are outside the linear range of the assay. Use a single type of reagent kit, standard and analyser within the laboratory, as the results may not be easily interchangeable between methods.

\section{PT-derived (PT-Fg) tests}

Fibrinogen estimations derived from the prothrombin time (PT-Fg) have been widely used in recent years (Vermylen 
et al, 1963; Natelson \& Dooley, 1974; Becker et al, 1984; Cambas et al, 1985; Chantarangkul et al, 1987; de Metz \& van Wersch, 1987; Hoffmann \& Verhappen, 1988; Rossi et al, 1988). The analyser is calibrated by performing a prothrombin time on a plasma (or series of plasma dilutions) of known fibrinogen concentration and by plotting a graph of the optical change against fibrinogen concentration. The optical change in each test sample is then converted to a fibrinogen value. As the technique is an indirect measurement of fibrinogen, the choice of calibrant or standard is very important. The attraction of the PT-Fg method is that it rapidly provides a fibrinogen value at no extra cost (other than that of the PT reagents), and it is currently used in approximately $50 \%$ of UK Haematology laboratories [UK National External Quality Assessment Scheme (NEQAS) for Blood Coagulation, 2000], although there is controversy regarding the suitability for clinical use. PT-Fg assays are really a collection of associated methods that vary depending on the method of calibration, type of analyser and reagent, and optical clarity of calibrant and test plasmas, as well as the nature of any coagulopathy present.

Calibration methods recommended by manufacturers differ; they may involve single- or multiple-point calibration, and the use of fresh normal pooled plasma of good optical quality or lyophilized plasma with a PT-Fg-specific calibration value. It is difficult to achieve calibration in some PT-Fg methods if the reference plasma has a high fibrinogen level or excess turbidity (MDA, 1999). Reference and test plasmas with high turbidity tend to show an increased discrepancy between PT-Fg and Clauss assays (Mackie et al, 2002).

Some, if not all, PT-Fg methods give higher values than the Clauss technique (Palareti et al, 1991; Chantarangkul et al, 1994; Chitolie et al, 1994, 1998; Kitchen et al, 1995; Lawrie et al, 1998; MDA, 2000; Mackie et al, 2002). This phenomenon has been observed in samples from patients with DIC, liver disease, renal disease, dysfibrinogenaemia, high fibrinogen levels and in those receiving anticoagulants, or thrombolytic therapy. In clinical samples, the discrepancy is not consistent over the entire range of observed fibrinogen values and may depend on the nature of the clinical group as well as the thromboplastin reagent used. Some laboratories have found apparently normal fibrinogen levels by PT-Fg, but reduced results by the Clauss assay in patients with dysfibrinogenaemia or those receiving thrombolytic therapy. In these groups, it is unclear which method correlates best with in vivo haemostasis. Other workers have found a good comparability between Clauss and particular types of PT-Fg assays (Rossi et al, 1988; de Cristofaro \& Landolfi, 1998); however, the latter stated that precision was inadequate in PT-Fg assays if either the PT was prolonged (e.g. as a result of anticoagulants) or the fibrinogen level was high.

Recent multicentre studies (MDA, 1999, 2000; Mackie et al, 2002) have shown that PT-Fg results vary according to the reagent and analyser (even between different models from the same manufacturer). A higher level of imprecision (CV typically $6-12 \%$ for normal plasma) was seen compared with Clauss assays, particularly if turbid reagents and reference plasmas were used. In thrombolytic therapy, fibrinogen degradation products (which were formed in excess of fibrin split products) interfered in the Clauss assay, but not in PT-Fg. Degraded fibrinogen molecules may reduce fibrin polymerization by termination of the fibrin chain, as they retain at least one fibrinopeptide A domain (Nieuwenhuizen \& Bos, 1999). The resulting weak, diffuse clot formation and delayed clot detection would affect the Clauss technique, while a large opacity change would still eventually occur, giving a higher PT-Fg level. This effect would be accentuated as the fibrinogen level fell, and by the dilution effect seen in Clauss assays. Intermediate and late FDPs cause a dose-dependent decrease in Clauss fibrinogen and an increase in PT-Fg (Oosting \& Hoffmann, 1997). More turbid fibrin gels are generated at lower thrombin concentrations (de Cristofaro \& Landolfi, 1998). As the thrombin concentration generated in the PT-Fg method is substantially lower (on a $\mathrm{U} / \mathrm{ml}$ basis) than that typically used in the Clauss assay, while the fibrinogen concentration is 2.5-5-fold higher, the fibrin gels generated by the PT-Fg method will be more turbid. This may particularly influence the results under conditions of abnormal fibrin polymerization.

PT-Fg results on clinical samples, particularly those with abnormal fibrinogen molecules and either high or low fibrinogen levels, show a sigmoid relationship with Clauss and other functional assays (Chitolie et al, 1998; MDA, 1999, 2000; Mackie et al, 2002). The plateau of the sigmoid curve differs depending on the PT-Fg thromboplastin reagent, reducing the upper limit of detection by as much as $2 \mathrm{~g} / \mathrm{l}$ (at equivalent Clauss values of 5-6 g/l). It is not known whether the upper cut-off limits vary between all reagents or whether there is a significant batch dependency. This problem precludes the use of simple data correction (algorithm) equations to normalize the PT-Fg results to Clauss values.

\section{Recommendations}

PT-Fg assays are not recommended for general use in Haematology laboratories. As both the analyser and reagent influence PT-Fg values, each reagent/instrument combination must be regarded as a separate assay type. The results from different instruments or thromboplastin reagents should not be mixed and separate reference ranges are required. PT-Fg assays are only suitable for samples with good optical clarity, where a normal fibrinogen value can be predicted, such as large epidemiological studies of normal populations, where an accurate measurement of either normal or elevated fibrinogen might not be required. In this setting, as well as external quality assessment exercises, it is important that data from different PT-Fg assays are not pooled. Where PT-Fg assays have been used, there may be difficulty in the subsequent interpretation of fibrinogen results from patient records, as the results are not interchangeable between laboratories or hospitals because of the differences between instrumentation and reagents. The reference plasma must be carefully selected for optical clarity, and calibration values must be available in relation to the International Standard (either directly or indirectly). Where necessary, local plasma pools should be calibrated 
against the International Standard in a Clauss assay and the observed value should be used in the PT-Fg calibration procedure.

\section{Clottable protein}

These assays are very accurate and have been used as reference assays for fibrinogen. Thrombin is added to plasma, in the absence of calcium ions, the clot is washed and then dissolved in alkaline urea or other reagents, followed by a spectrophotometric protein assay or estimation (Ratnoff \& Menzie, 1951; Jacobsson, 1955; Blomback \& Blomback, 1956; Swain \& Feders, 1967; Gaffney \& Wong, 1992). As the majority of the protein in the clot will be fibrin, the protein concentration will be approximately equivalent to the fibrinogen concentration. One convenient method (Blomback \& Blomback, 1956) is to measure the absorbance at $282 \mathrm{~nm}$ and to calculate the fibrinogen concentration using the extinction coefficient. Clottable protein assays are time consuming, labour intensive and technically difficult, making them unsuitable for routine use in coagulopathy screening. However, they can be of occasional use in the investigation of congenital fibrinogen defects (Table III).

\section{Immunological assay}

A variety of immunological assays are available: enzymelinked immunosorbent assays (ELISAs), radial immunodiffusion and electrophoretic techniques, take many hours to perform, the former giving the best accuracy and precision. Some clinical analysers (e.g. BN-100; Dade Behring, Marburg, Germany) have a facility for fibrinogen assay using immunonephelometry. All available immunological assays have the disadvantage of measuring protein concentration rather than functional activity. False estimates may be obtained in some methods where degraded forms of fibrinogen are present, as these have a different antigenicity and may migrate at different rates in immunodiffusion and electrophoresis techniques. These effects are likely to explain the discrepancies between fibrinogen antigen and either Clauss or PT-Fg results observed in certain clinical groups.

Some commercial assays utilize monoclonal antibodies directed against terminal regions of the fibrinogen molecule; these allow the detection of intact (non-proteolysed) fibrinogen or various specific types of degraded fibrinogen molecule. Such assays are sometimes useful in research studies or specialized investigations of individual patients.

Table III. The recommended types of fibrinogen assay in different clinical situations.

Bleeding diathesis

Clauss

Congenital fibrinogen Clauss + clottable protein + immunoassay defects

DIC, acquired defects Clauss

High fibrinogen levels Clauss or immunoassay

Thrombolytic therapy Clauss

\section{Recommendations}

ELISAs and immunonephelometric assays give comparable results overall, although the latter has a high degree of variability.

The Second International Standard for fibrinogen has been calibrated by functional rather than immunological methods. Until a directly calibrated standard becomes available, the same standard should be used for both types of fibrinogen assay and similar potency assumed. It is essential that the same standard material be used for different fibrinogen assays within an individual laboratory, to avoid systematic error.

Fibrinogen antigen measurement is suitable for studies assessing fibrinogen as a risk factor for cardiovascular disease (Cremer et al, 1994; Sweetnam et al, 1996, 1998), where normal or elevated fibrinogen levels are anticipated. It also has utility in the investigation and differentiation of congenital fibrinogen defects (Table III).

\section{Other methods}

The fibrinogen titre is an emergency test where plasma (and, therefore, fibrinogen) is diluted in different types of buffer, before the addition of thrombin (Mackie \& Machin, 1989). The titre is reported as the last dilution to display a clot after a given incubation time. The test can be performed in the presence of various inhibitors, such as epsilon-amino$\mathrm{N}$-caproic acid (EACA), to block plasmin, and protamine to block heparin and FDP's. However, the results are very inaccurate, and the test is time consuming and does not lend itself to automation. Owing to the widespread availability of automated analysers, the test has virtually disappeared from clinical laboratories and it is not recommended.

In gravimetric assays (Mackie \& Machin, 1989), fibrinogen is clotted from dilute plasma, using a strong thrombin solution and calcium chloride. The clot is compressed to express solution and non-clotted proteins, washed in saline, and then dried and weighed on an accurate microbalance (the number of available decimal places on the microbalance obviously affects accuracy). The assay demands a high degree of technical ability to obtain reproducible results and is time consuming, as the clot must be completely dry before weighing. It is not, therefore, of clinical utility when fibrinogen assays are required urgently. The method does not lend itself to large numbers of tests, cannot be automated and is not recommended for routine use.

Sulphite precipitation techniques (Rampling \& Gaffney, 1976) have been described, but are unreliable in some clinical situations (particularly in patients with acute phase reaction), and are not normally used in hospital haematology laboratories.

\section{PRE-TEST VARIABLES}

The main pretest variables affecting fibrinogen assays are summarized in Table IV. The recommended anticoagulant for fibrinogen assay is tri-sodium citrate (World Health Organization, 1999), at a strength of $0 \cdot 105-0 \cdot 109 \mathrm{~mol} / \mathrm{l}$ 
Table IV. Pre-test variables.

Anticoagulant
Correct filling
Haematocrit
Venepuncture
Blood and plasma inspection
Platelet depletion
Storage
Freezing and thawing
Correct dilution

Anticoagulant

Blood and plasma inspection

Storage

Correct dilution

\author{
Tri-sodium citrate $(0 \cdot 105-0 \cdot 109 \mathrm{~mol} / \mathrm{l})$ \\ 9 parts blood to 1 part citrate \\ Adjust citrate for polycythaemia \\ Clean, fast, minimal stasis \\ Clots, haemolysis, lipaemia, icterus \\ Centrifuge $>1700 \mathrm{~g}, 10 \mathrm{~min},<10 \times 10^{9} / \mathrm{l}$ \\ $4^{\circ} \mathrm{C}$ for $48 \mathrm{~h}$ (unless DIC); $-70^{\circ} \mathrm{C}$ for $\geq 18$ months \\ Thaw at $37^{\circ} \mathrm{C}$ for $\geq 5 \mathrm{~min}$, mix well \\ Dilute concentrates and plasmas \\ with high fibrinogen, so that values are \\ within standard curve limits
}

(1 part anticoagulant: 9 parts blood), as used for other blood coagulation tests (NCCLS, 1998). Inadequate filling of the collection tube affects this ratio and leads to inaccurate results. For haematocrits greater than $0.55 \mathrm{l} / \mathrm{l}$, the final blood citrate concentration requires adjustment and charts are available which indicate the amounts of anticoagulant and blood to be mixed (NCCLS, 1998). It is essential that blood is collected by clean venepuncture, with minimal stasis, and as rapidly as possible (Rosenson et al, 1998). Prior to fibrinogen determination, blood samples should be inspected by gentle inversion for the presence of clots. Samples with any evidence of clots or marked haemolysis should be rejected.

Plasma should be prepared so that platelets are removed (to a count of $<10 \times 10^{9} / \mathrm{l}$ ) by centrifugation at a minimum of $1700 \mathrm{~g}$ for $10 \mathrm{~min}$; this can be performed at room temperature. Severely lipaemic or icteric samples may cause analysis difficulties in some photo-optical methods of fibrinogen measurement. Samples can be stored at room temperature prior to fibrinogen determination, for at least $4 \mathrm{~h}$ and probably longer. If more prolonged storage is required, then $4^{\circ} \mathrm{C}$ is a suitable temperature for Clauss fibrinogen determination, and storage at $4^{\circ} \mathrm{C}$ for up to $48 \mathrm{~h}$ did not affect assay results in one study (Rosenson et al, 1998). Plasma can be stored deep frozen for longer periods (Cushman et al, 1995), preferably at $-70^{\circ} \mathrm{C}$, where it is stable for at least 18 months (Blanco et al, 2000). Storage of samples from patients with DIC or those receiving thrombolytic therapy may lead to in vitro proteolysis of fibrinogen unless suitable protease inhibitors are included in the anticoagulant. If plasma is frozen prior to analysis, it should be immediately transferred to a $37^{\circ} \mathrm{C}$ water bath, thawed for $5 \mathrm{~min}$ at $37^{\circ} \mathrm{C}$ and mixed by inversion prior to analysis. Any slow or partial thaw can lead to the formation of cryo-precipitate, which contains fibrinogen, and thus may affect the results. For assay of fibrinogen in therapeutic cryo-precipitate products and other fibrinogen concentrates, a full thaw at $37^{\circ} \mathrm{C}$ is necessary followed by careful mixing to re-suspend the fibrinogen. This material will normally require a greater dilution than normal plasma in order to achieve a concentration that can be successfully assayed by the Clauss technique.

\section{STANDARDS, REFERENCE PREPARATIONS AND REFERENCE RANGES}

Local reference plasmas may be prepared by pooling plasmas from at least six healthy normal donors and calibrating against National (British Standard for Blood Coagulation Factors, Plasma, NIBSC) or International (Second International Standard for Fibrinogen, Plasma) standards. These pools should ideally be stored in aliquots at $-70^{\circ} \mathrm{C}$ and completely thawed at $37^{\circ} \mathrm{C}$ before use. If commercial reference preparations are used, they should be suitably calibrated; some have shown significant deviations from the International Standard in their potency estimates (MDA, 1999; Mackie et al, 2002). If commercial plasmas are used, it is advisable to select preparations with a good optical clarity wherever possible.

In order to provide reference materials for widespread use, lyophilization is required to prevent the deterioration of fibrinogen. Reference plasmas should not be turbid, as this creates practical problems with the calibration of photooptical coagulometers for Clauss and PT-Fg assays. High fibrinogen concentrations may be incompatible with PT-Fg calibrations when using certain analysers and reagents. Purified fibrinogen is generally unsuitable in the preparation of standard materials for assay in clinical laboratories, as after its lyophilization there are often problems with solubility, specific activity, degradation and optical clarity.

Reference ranges should be established in each laboratory, using at least 40 healthy normal subjects, covering a wide age range and both sexes. Blood samples from patients should not be used, even if they are free from any major illness, as it is likely that they will be stressed or may have an acute phase reaction, which would skew the range of values.

\section{QUALITY CONTROL}

It is recommended that all laboratories participate in an accredited external quality assessment scheme and investigate any evidence of local problems. For participants in larger groups, it may be possible to identify methodological bias related to particular components of the assay (such as reference plasma or source of reagent) and these should be 
taken into account when selecting a suitably standardized technique.

An internal quality control (QC) sample, with a normal fibrinogen level, should be analysed with each group of fibrinogen determinations (or at intervals of 2-3 $\mathrm{h}$ for continuous processing systems). A QC sample with reduced or raised fibrinogen may be useful, depending on the purpose for which fibrinogen assays are being performed, or if unexpected results are obtained from test samples. A written record of internal QC results should be maintained to include the result, time of analysis and reagent batch/lot numbers. It is useful to maintain a cumulative record, preferably as a graph, because this can identify any gradual drift in QC results, which could indicate that the method is moving out of the range of the control. If a $\mathrm{QC}$ result lies outside acceptable limits (frequently provided by manufacturers as a target range), then patient testing must be suspended until the problem has been identified and resolved.

\section{AUDIT}

Routine audit of the laboratory measurement and the clinical utility of fibrinogen assays should be incorporated into routine practice. The potential variability of laboratory methodology, involving both reagents and end-point detection systems, should be regularly examined by participation in an accredited external quality assessment scheme. Clinical audit is more complex and should involve the diagnostic value of a low/normal fibrinogen level in the investigation of acquired and inherited bleeding disorders, and also the value of normal/high levels in the risk assessment of thrombotic cardiovascular disease. For the investigation of bleeding disorders, a comparison of fibrinogen levels with the thrombin time, the variation during thrombolytic therapy and the correlation with replacement therapy (i.e. fibrinogen concentrates) should be studied. Specific levels of fibrinogen should be defined for further action, as part of clinical protocols (i.e. the levels following massive transfusion or acute DIC when specific replacement therapy must be initiated). Risk assessment of arterial thrombosis is more controversial, but levels of risk in specific diseases (i.e. myocardial infarction) need to be defined with actual fibrinogen levels divided into quartiles (or even quintiles) of the overall normal range. These ranges could then be discussed with the relevant clinicians, so that patients may be counselled as to their overall risk, specific interventions advised (such as stopping smoking), and the timing of follow-up measurements decided. To differentiate high levels from an acute phase response, comparisons with other acute phase proteins, particularly C-reactive protein and von Willebrand factor antigen, can be studied.

\section{CONCLUSIONS}

The Clauss fibrinogen assay appears to be the most reliable method for general use in clinical laboratories. In the UK, most clinical experience with fibrinogen levels in coagulo-

pathies relates to the Clauss assay and most educational material is also based on this technique. The PT-Fg method and immunological techniques may be useful in large population studies where elevated fibrinogen is sought, but may not always provide accurate fibrinogen measurements. For the investigation of congenital dysfibrinogenaemias, both functional (Clauss or clottable protein assays) and immunological fibrinogen assays should be performed (Haverkate \& Samama, 1995) to identify any discrepancy between results.

PT-Fg levels appear to show a false elevation in a variety of clinical settings and, if the assay is used, it must be supported by a different functional assay before either diagnosis or management decisions are made. In light of the available literature, which shows that the PT-Fg measurement is significantly influenced by the choice of calibrant, reagent and analyser, the test is not recommended for routine clinical use.

\section{EXPIRY: DECEMBER 2006; REVIEW: DECEMBER} 2005

\section{ACKNOWLEDGMENTS}

The authors and Task Force wish to acknowledge the helpful and constructive comments on this manuscript made by members of the British Society for Haematology.

\section{DISCLAIMER}

Although the advice and information contained in these guidelines is believed to be true and accurate at the time of going to press, neither the authors nor the publishers can accept any legal responsibility for any errors or omissions that may have been made.

\author{
${ }^{1}$ Haematology Department, \\ University College London, \\ ${ }^{2}$ Sheffield Haemophilia and \\ Thrombosis Centre \\ Laboratory, Royal \\ Hallamshire Hospital, \\ Sheffield, and ${ }^{3}$ Department \\ of Medicine, University of \\ Glasgow, Glasgow, UK
}

\author{
IAN J. MACKIE ${ }^{1}$ \\ SteVEN Kitchen $^{2}$ \\ SAMUEL J. MACHIN ${ }^{1}$ \\ GORDON D. O. LOWE \\ ON BEHALF OF \\ THE HAEMOSTASIS \\ AND Thrombosis \\ TAsk Force of the \\ British Committee \\ FOR STANDARDS \\ in HaEmatology
}

\section{REFERENCES}

Becker, U., Bartl, K. \& Wahlefeld, A.W. (1984) A functional photometric assay for plasma fibrinogen. Thrombosis Research, 35 , 475-484.

Blanco, M., Colesse, G., Woodhams, B. \& Gourmelin, Y. (2000) Stability of coagulation factors in frozen plasma. Haemostasis, 30, 37-38a.

Blomback, B. \& Blomback, M. (1956) Preparation of human fibrinogen fraction 1-2. Arkiv for Kemi, 10, 415-443. 
van der Bom, J.G., de Maat, M.P.M., Bots, M.L., Hofman, A., Kluft, C. \& Grobbee, D.E. (1997) Seasonal variation in fibrinogen in the Rotterdam Study. Thrombosis and Haemostasis, 78, 1059-1062.

Cambas, J.P., Bierme, R., Martinon, J.C. \& Dousset, B. (1985) Evaluation des performances d'un automate en coagulation: l'Electra 700. Nouvelle Revue Francaise D'hematologie, 25, 313-320.

Chantarangkul, V., Tripodi, A. \& Mannucci, P.M. (1987) Evaluation of a fully automated centrifugal analyzer for performance of hemostasis tests. Clinical Chemistry, 33, 1888-1890.

Chantarangkul, V., Tripodi, A. \& Mannucci, P.M. (1994) Results of a collaborative study for fibrinogen measurement. Evidence that the use of a common calibrator improves interlaboratory agreement. Blood Coagulation and Fibrinolysis, 5, 761-766.

Chitolie, A., Mackie, I.J., Grant, D., Hamilton, J.L. \& Machin, S.J. (1994) Inaccuracy of the 'derive' fibrinogen measurement. Blood Coagulation and Fibrinolysis, 5, 955-957.

Chitolie, A., Mackie, I.J. \& Machin, S.J. (1998) The type of thromboplastin reagent has important effects on the PT-derived fibrinogen potency. Laboratory Hematology, 4, 149-155.

Clauss, A. (1957) Gerinnungsphysiologische schnellmethode zur bestimmung des fibrinogens. Acta Haematologica, 17, 237-246.

Cremer, P., Nagel, D., Labrot, B., Mann, H., Muche, R., Elster, H. \& Seidel, C. (1994) Lipoprotein $\mathrm{Lp}(\mathrm{a})$ as predictor of myocardial infarction in comparison to fibrinogen, LDL cholesterol and other risk factors: results from the prospective Gottingen Risk Incidence and Prevalence Study (GRIPS). European Journal of Clinical Investigation, 24, 444-453.

de Cristofaro, R. \& Landolfi, R. (1998) Measurement of plasma fibrinogen concentration by the prothrombin-time-derived method: applicability and limitations. Blood Coagulation and Fibrinolysis, 9, 251-259.

Cushman, M., Cornell, E.S., Howard, P.R., Bovill, E.G. \& Tracy, R.P. (1995) Laboratory methods and quality assurance in the cardiovascular health study. Clinical Chemistry, 41, 264-270.

Eliasson, M., Evrin, P.E., Lundblad, D., Asplund, K. \& Ranby, M. (1993) Influence of gender, age and sampling time on plasma fibrinolytic variables and fibrinogen. A population study. Fibrinolysis, 7, 1-8.

Gaffney, P.J. \& Wong, M.Y. (1992) Collaborative study of a proposed international standard for plasma fibrinogen measurement. Thrombosis and Haemostasis, 68, 428-432.

Hantgan, R.R., Francis, C.W. \& Marder, V.J. (1994) Fibrinogen structure and physiology. In: Hemostasis and Thrombosis: Basic Principles \& Clinical Practice (ed. by R.W. Colman, J. Hirsh, V.J. Marder \& E.W. Salzman), pp. 277-300. JB Lippincott, Philadelphia.

Haverkate, F. \& Samama, M. (1995) Familial dysfibrinogenemia and thrombophilia. Report on a study of the SSC subcommittee on fibrinogen. Thrombosis and Haemostasis, 73, 151-161.

Hoffmann, J.J.M.L. \& Verhappen, M.A.L. (1988) Automated nephelometry of fibrinogen: analytical performance and observations during thrombolytic therapy. Clinical Chemistry, 34, $2135-2140$

Humphries, S.E., Henry, J.A. \& Montgomery, H.E. (1999) Geneenvironment interaction in the determination of levels of haemostatic variables involved in thrombosis and fibrinolysis. Blood Coagulation and Fibrinolysis, 10, s17-s21.

Jacobsson, K. (1955) Studies in the determination of fibrinogen in human blood plasma. Scandinavian Journal of Clinical Laboratory Investigation, 7, 1-54.

Kitchen, S., Jennings, I. \& Preston, F.E. (1995) Comparison of fibrinogen determinations using a Clauss assay and two pro- thrombin time derived methods. Thrombosis and Haemostasis, 73 , $1245 \mathrm{a}$.

Kluft, C. \& Lansink, M. (1997) Effect of oral contraceptives on haemostasis variables. Thrombosis and Haemostasis, 78, 315-326.

Lawrie, A.S., McDonald, S.J., Purdy, G., Mackie, I.J. \& Machin, S.J. (1998) Prothrombin time derived fibrinogen determination on the Sysmex CA-6000 ${ }^{\mathrm{TM}}$. Journal of Clinical Pathology, 51, 462466.

Lowe, G.D.O. \& Rumley, A. (1999) Use of fibrinogen and fibrin Ddimer in prediction of arterial thrombotic events. Thrombosis and Haemostasis, 82, 667-672.

Lowe, G.D.O., Rumley, A., Woodward, M., Morrison, C.E., Philippou, H., Lane, D.A. \& Tunstall-Pedoe, H. (1997) Epidemiology of coagulation factors, inhibitors and activation markers: The third Glasgow MONICA survey. I. Illustrative reference ranges by age, sex and hormone use. British Journal of Haematology, 97, 775-784.

Ludlam, C.A., Bennett, B., Fox, K.A.A., Lowe, G.D.O. \& Reid, A.W (1995) Guidelines for the use of thrombolytic therapy. Blood Coagulation and Fibrinolysis, 6, 273-285.

de Maat, M.P.M., Lowe, G.D.O. \& Haverkate, F. (1999) Fibrinogen. In: Laboratory Techniques in Thrombosis: A Manual (ed. by J. Jespersen, R.M. Bertina \& F. Haverkate), pp. 79-88. Kluwer Academic Publishers, Dordrecht.

Machin, S.J. \& Mackie, I.J. (1993) Routine measurement of fibrinogen concentration: not clinically feasible. British Medical Journal, 307, 882-883.

Mackie, I.J. \& Machin, S.J. (1989) Laboratory Haematology: An Account of Laboratory Techniques (ed. by I. Chanarin), pp. 279 280. Churchill Livingstone, Edinburgh.

Mackie, I.J., Lawrie, A.S., Kitchen, S., Gaffney, P.J., Howarth, D., Lowe, G.D.O., Martin, J., Purdy, G., Rigsby, P. \& Rumley, A. (2002) A performance evaluation of commercial fibrinogen reference preparations and assays for Clauss and PT-derived fibrinogen. Thrombosis and Haemostasis, 87, 997-1005.

Marder, V.J. \& Sherry, S. (1988) Thrombolytic therapy: current status. New England Journal of Medicine, 318, 1512-1520, 15851595.

MDA (1999) Medical Devices Agency Evaluation Report: Fibrinogen Standards and Reference preparations. MDA 99/47 ISBN 184182 0784. Her Majesty's Stationery Office, Norwich.

MDA (2000) Medical Devices Agency Evaluation Report: Fibrinogen Assay reagents and Methods. MDA 2000/01 ISBN 184182 139X. Her Majesty's Stationery Office, Norwich.

de Metz, M. \& van Wersch, J.W.J. (1987) Use of a centrifugal analyzer for a chromogenic prothrombin time, a chromogenic activated partial thromboplastin time and a kinetic fibrinogen assay in a routine hospital laboratory. Haemostasis, 17, 254259.

Natelson, E.A. \& Dooley, D.F. (1974) Rapid determination of fibrinogen by thrombokinetics. American Journal of Clinical Pathology, 61, 828-833.

NCCLS (1998) Collection, Transport and Processing of Blood Specimens for Coagulation Testing and General Performance of Coagulation assays: Approved Guideline 3rd edn. NCCLS, H21-A3, 18, number 20.

Nieuwenhuizen, W. \& Bos, R. (1999) Soluble fibrin and degradation products of fibrinogen (FgDP), fibrin (FbDP; D-dimer) and total of FgDP and FbDP (TDP). In: Laboratory Techniques in Thrombosis: A Manual (ed. by J. Jespersen, R.M. Bertina \& F. Haverkate), pp. 275-284. Kluwer Academic Publishers, Dordrecht.

Oosting, J.D. \& Hoffmann, J.J.M.L. (1997) Evaluation of an automated photometric fibrinogen assay. Blood Coagulation and Fibrinolysis, 8, 321-326. 
Palareti, G., Maccaferri, M., Manotti, C., Tripodi, A., Chantarangkul, V., Rodeghiero, F., Ruggeri, M. \& Mannucci, P.M. (1991) Fibrinogen assays: a collaborative study of six different methods. C.I.S.M.E.L Comitaro Italiano Per la Standardizzione Dei Methodi in Ematologia E Labatorio, 37, 714-719.

Rampling, M.W. \& Gaffney, P.J. (1976) Measurement of fibrinogen in plasma. In: Progress in Chemical Fibrinolysis \& Thrombosis (ed. by J.F. Davidson, M.M. Samama \& P.C. Desnoyers), Vol. 2, pp. 91-105. Raven Press, New York.

Ratnoff, O.D. \& Menzie, C. (1951) A new method for the determination of fibrinogen in small samples of plasma. Journal of Laboratory and Clinical Medicine, 37, 316-320.

Rosenson, R.S., Staffileno, B.A. \& Tangney, C.C. (1998) Effects of tourniquet technique, order of draw, and sample store on plasma fibrinogen. Clinical Chemistry, 44, 688-690.

Rossi, E., Mondonico, P., Lombardi, A. \& Preda, L. (1988) Method for determination of functional (clottable) fibrinogen by the new family of ACL coagulometers. Thrombosis Research, 52, $453-468$

Swain, W. \& Feders, M.B. (1967) Fibrinogen assay. Clinical Chemistry, 13, 1026-1028.

Sweetnam, P.M., Thomas, H.F., Yarnell, J.W.G., Beswich, A., Baker, I.A. \& Elwood, P.C. (1996) Fibrinogen, viscosity and the 10-year incidence of ischaemic heart disease. The Caerphilly and Speedwell studies. European Heart Journal, 17, 18141820 .
Sweetnam, P.M., Yarnell, J.W.G., Lowe, G.D.O., Baker, I.A., O'Brien, J.R., Rumley, A., Etherington, M.D., Whitehead, P.J. \& Elwood, P.C. (1998) The relative predictive power of heat-precipitation nephelometric and clottable (Clauss) fibrinogen in the prediction of ischaemic heart disease: the Caerphilly and Speedwell studies. British Journal of Haematology, 100, 582-588.

Thompson, S.G., Martin, J.C. \& Meade, T.W. (1987) Sources of variability in coagulation factor assays. Thrombosis and Haemostasis, 58, 1073-1077.

UK National External Quality Assessment Scheme (NEQAS) for Blood Coagulation (2000) Report on Survey 120, Sheffield.

Vermylen, C., de Vreker, R.A. \& Verstraete, M. (1963) A rapid enzymatic method for assay of fibrinogen fibrin polymerization time (FPT test). Clinica Chimica Acta, 8, 418-424.

Whicher, J.T. \& Dieppe, P.A. (1985) Acute phase proteins. Clinical Immunology and Allergy, 5, 425-446.

World Health Organisation Report (1999) Use of Anticoagulants in Diagnostic Laboratory Investigations. World Health Organisation/DIL/LAB/99, 1 Rev 1.

Keywords: fibrinogen assays, Clauss assay, PT-derived fibrinogen (PT-Fg) tests, fibrinogen antigen. 